\title{
Long-Lived Termite Queens Exhibit High Cu/Zn-Superoxide Dismutase Activity
}

\author{
Eisuke Tasaki $\mathbb{D}^{1,2,3}$ Kazuya Kobayashi, ${ }^{4}$ Kenji Matsuura, ${ }^{1}$ and Yoshihito Iuchi $\mathbb{D}^{2,3,5}$ \\ ${ }^{1}$ Laboratory of Insect Ecology, Graduate School of Agriculture, Kyoto University, Kitashirakawa Oiwakecho, Kyoto 606-8502, Japan \\ ${ }^{2}$ Department of Applied Bioresources Chemistry, The United Graduate School of Agriculture, Tottori University, 4-101 Koyamacho- \\ minami, Tottori 680-8553, Japan \\ ${ }^{3}$ Department of Biological Chemistry, Faculty of Agriculture, Yamaguchi University, 1677-1 Yoshida, Yamaguchi 753-8515, Japan \\ ${ }^{4}$ Hokkaido Forest Research Station, Field Science Education and Research Center, Kyoto University, 553 Tawa Shibecha-cho \\ Kawakami-gun, Hokkaido 088-2339, Japan \\ ${ }^{5}$ Graduate School of Sciences and Technology for Innovation, Yamaguchi University, 1677-1 Yoshida, Yamaguchi 753-8515, Japan
}

Correspondence should be addressed to Yoshihito Iuchi; yiuchi@yamaguchi-u.ac.jp

Received 22 November 2017; Accepted 8 January 2018; Published 13 February 2018

Academic Editor: Janusz Gebicki

Copyright (C) 2018 Eisuke Tasaki et al. This is an open access article distributed under the Creative Commons Attribution License, which permits unrestricted use, distribution, and reproduction in any medium, provided the original work is properly cited.

\begin{abstract}
In most organisms, superoxide dismutases (SODs) are among the most effective antioxidant enzymes that regulate the reactive oxygen species (ROS) generated by oxidative energy metabolism. ROS are considered main proximate causes of aging. However, it remains unclear if SOD activities are associated with organismal longevity. The queens of eusocial insects, such as termites, ants, and honeybees, exhibit extraordinary longevity in comparison with the nonreproductive castes, such as workers. Therefore, the queens are promising candidates to study the underlying mechanisms of aging. Here, we found that queens have higher $\mathrm{Cu} /$ $\mathrm{Zn}$-SOD activity than nonreproductive individuals of the termite Reticulitermes speratus. We identified three $\mathrm{Cu} / \mathrm{Zn}-\mathrm{SOD}$ sequences and one Mn-SOD sequence by RNA sequencing in $R$. speratus. Although the queens showed higher Cu/Zn-SOD activity than the nonreproductive individuals, there were no differences in their expression levels of the $\mathrm{Cu} / \mathrm{Zn}-\mathrm{SOD}$ genes RsSOD1 and RsSOD3A. Copper $\left(\mathrm{Cu}^{2+}\right.$ and $\left.\mathrm{Cu}^{+}\right)$is an essential cofactor for $\mathrm{Cu} / \mathrm{Zn}-\mathrm{SOD}$ enzyme activity, and the queens had higher concentrations of copper than the workers. These results suggest that the high $\mathrm{Cu} / \mathrm{Zn}$-SOD activity of termite queens is related to their high levels of the cofactor rather than gene expression. This study highlights that Cu/Zn-SOD activity contributes to extraordinary longevity in termites.
\end{abstract}

\section{Introduction}

Queens of termites, ants, and honeybees are representative eusocial insects that live up to 10 times longer than nonreproductive workers, although they possess the same genome [1-3]. Generally, longevity negatively correlates with reproduction among species [4]. Most animal species show a gradual decline in reproduction with age [5]. Indeed, sterile female flies have longer lifespans than fertile females [6], and germline ablation extends worm longevity [7]. However, the queens of eusocial insects are believed to live for long periods while also laying large numbers of eggs [8]. Because of their unique characteristics, eusocial insects are expected to facilitate the discovery of the mechanisms involved in longevity determination [9].

In most organisms, superoxide dismutases (SODs) are the first line of antioxidant defense against the toxic reactive oxygen species (ROS) that are generated by aerobic metabolism. SODs convert the toxic superoxide anion radicals into hydrogen peroxide, which is subsequently detoxified by catalase, glutathione peroxidase, and peroxiredoxin. At present, two types of $\mathrm{Cu} / \mathrm{Zn}-\mathrm{SOD}$, which are localized to either cytoplasmic compartments (SOD1) or extracellular elements (SOD3), and Mn-SOD (SOD2), which is exclusively located in mitochondria, have been identified in a variety of organisms $[10,11]$. The accumulation of ROS-mediated damage 


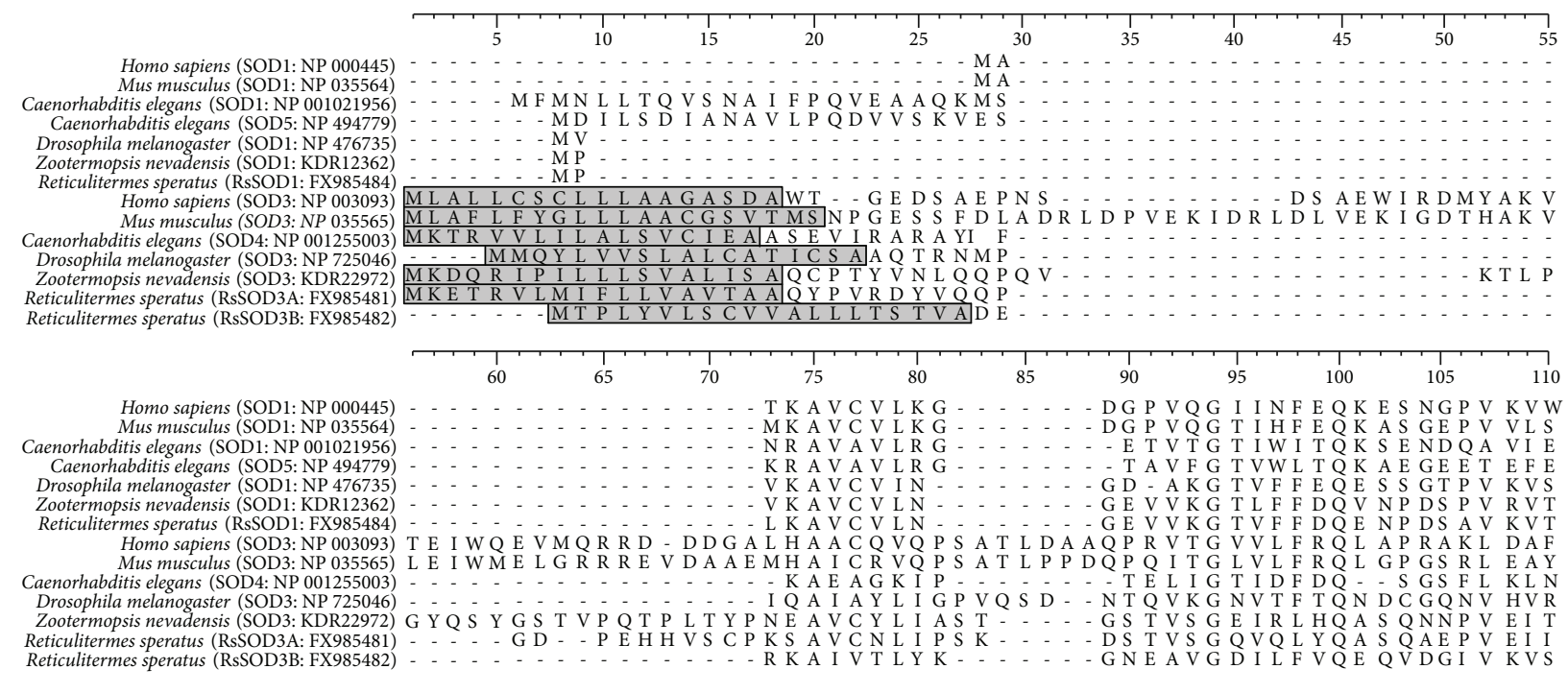

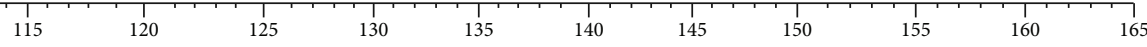

Homo sapiens (SOD1: NP 000445) G S I K G L T E G - - - L H G F HVHE F G D NTA G C T S A GP HF N P L S R K HG G P K D E E R HVG D Mus musculus (SOD1: NP 035564) GQ I T G L T E G - - - Q H G F HVHQY G D N T Q G C T S A G P H N P H S K K H G P A D E E H V D D Caenorhabditis elegans (SOD1: NP 001021956) GE I K G L T P G - - - L H G G H H HQ Y G D S T N G C I S A G P H F N P F G K T H G G P K S E I R H V G D

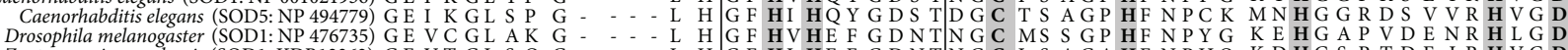

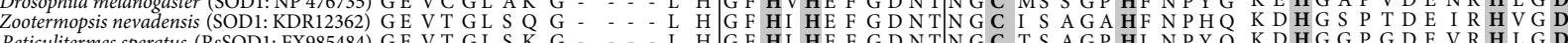
Reticulitermes speratus (RsSOD1: FX985484) GE V T G G S K G - - - - L H G G H I HE F G D N T N G C T S A G P H L N P Y Q K D H G G P G D E V R H L G D

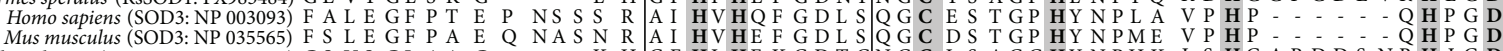
Caenorhabditis elegans (SOD4: NP 001255003) G S V S G L A A G - - K K H G F H I HE K G D T G N G C L S A G G H N P H K L S H G A P D D S N R H I G D

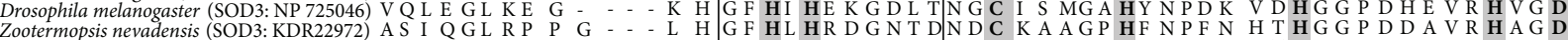
Zootermopsis nevadensis (SOD3: KDR22972) A S I Q G G R P P
Reticulitermes

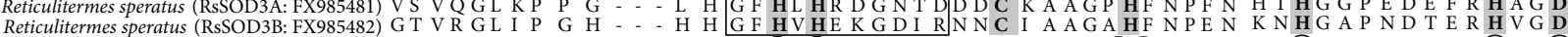

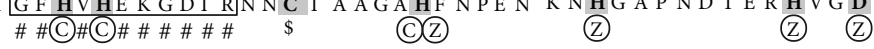

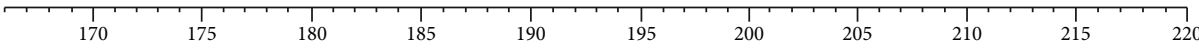

Homosapiens (SOD1: NP 000445) L G N V T A D K D G V A D V S I E D S V I S L S G D HC - - I I G R T L V V HE K A D D L G K G G N - - - E E Mus musculus (SOD1: NP 035564) L G N V T A G K D G V A N V S I E D R V I S L S G E H S - - I I GR T M V V HE KQDD L G K GG N - - - E E

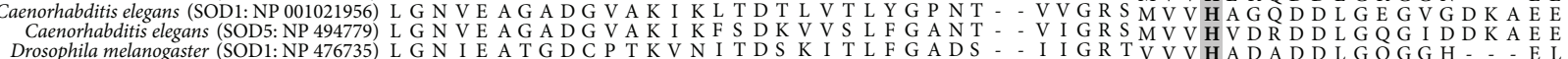

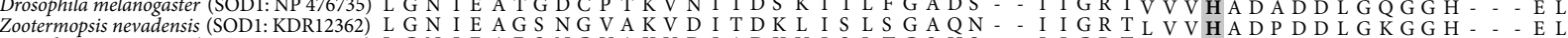
Zootermopsis nevadensis (SOD1: KDR12362) L G N I E A G S N G V A K V D I T D K L I S L S G A Q N - - I I G R T L V V H A D P D D L G K G G H - - - E L
Reticulitermes speratus (RsSOD1: FX985484) L G N I E A E S N G V A K V D I A D K V I S L T G S H S - - I I G R T L V V H A D P D D L G K G G H - - - E L

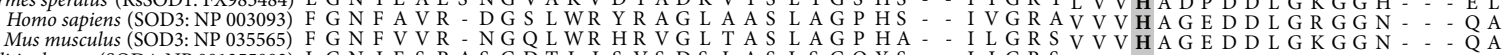

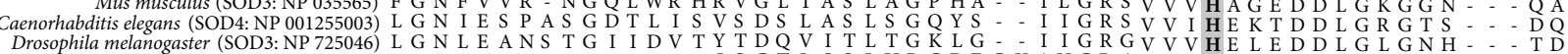

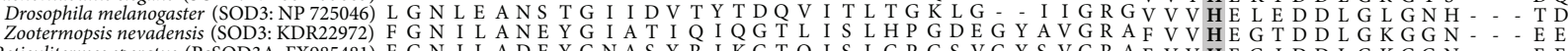

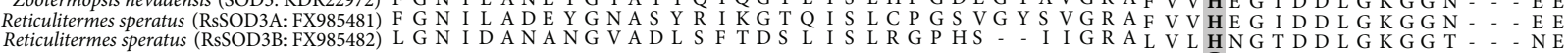

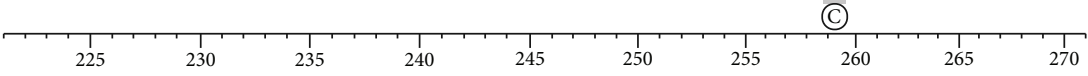

Homo sapiens (SOD1: NP 000445) S T K T G N A G S R L A C G V I G I A Q Caenorhabditis elegans (SOD1: NP 001021956) S K K T G N A G A R A A C G V I A L A A P Q Caenorhabditis elegans (SOD5: NP 494779) S L K K T G N A G A R A A C G V I A L A A P A Drosophila melanogaster (SOD1: NP 476735) S K S T G N A G A R I G C G V I G I A K V Zootermopsis nevadensis (SOD1: KDR12362) S K T T G A A G A R S A C G V V G I A K Reticulitermes speratus (RsSOD1: FX985484) S K T T T G N A A G A R S A C G V V G I A K Homo sapiens (SOD3: NP 003093) S V E N G N A G R R L A C C V V G V C G P G L W E R Q A R E H S E R K K R R R E S E C K A A -

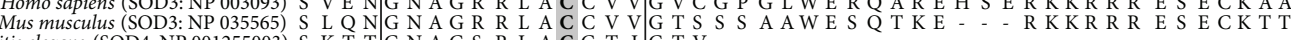

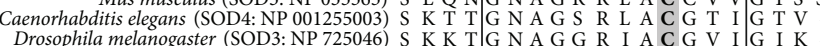
Drosophila melanogaster (SOD3: NP 725046)
Z S K K K T G N N A G G R I A C C G V I I G I K -

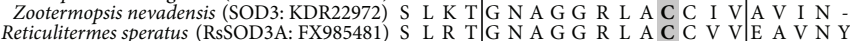

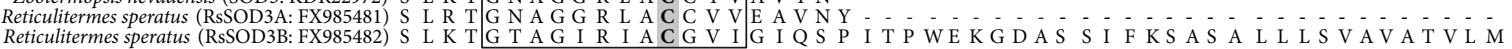

FIgURE 1: Alignment and structure conservation of $\mathrm{Cu} / \mathrm{Zn}-\mathrm{SOD}$ sequences. Multiple sequence alignment of Homo sapiens, Mus musculus, Caenorhabditis elegans, Drosophila melanogaster, Zootermopsis nevadensis, and Reticulitermes speratus. The signal cleavage site is indicated by a grey box. Conserved functional residues are highlighted in bold characters in grey (circled $\mathrm{C}=$ copper-binding site; circled $\mathrm{Z}=$ zincbinding site; circled $\mathrm{C}$, circled $\mathrm{Z}=$ copper- and zinc-binding sites; and $\$=$ intrasubunit disulphide bridge site). SOD signature sequences are denoted in blocks with \#.

is associated with aging and negative effects on longevity $[12,13]$. Therefore, SODs contribute to stress resistance associated with the lifespan of an organism. Indeed, many studies have reported that SODs contributed to the longevity of the fruit fly Drosophila melanogaster [14-16], the yeast Saccharomyces cerevisiae [17], and the nematode
Caenorhabditis elegans [18]. However, there are also many conflicting reports [19-22]. The impact of high SOD activity levels on longevity remains to be determined.

Unlike short-lived organisms, such as fruit flies, yeasts, and nematodes, termites sustain their maturity for a long time and sexually reproduce each year, which leads to an 
TABLE 1: Predicted location and signal cleavage site of putative SOD proteins.

\begin{tabular}{|c|c|c|}
\hline Gene sequence (database number) & Localization (reliability class) $^{\mathrm{a}}$ & $\overline{\text { Signal cleavage }}$ \\
\hline Homo sapiens (SOD1: NP 000445) & $-(2)$ & None \\
\hline Mus musculus (SOD1: NP 035564) & $-(2)$ & None \\
\hline Caenorhabditis elegans (SOD1: NP 001021956) & $-(4)$ & None \\
\hline Caenorhabditis elegans (SOD5: NP 494779) & $-(2)$ & None \\
\hline Drosophila melanogaster (SOD1: NP 476735) & $-(2)$ & None \\
\hline Zootermopsis nevadensis (SOD1: KDR12362) & $-(2)$ & None \\
\hline Reticulitermes speratus (RsSOD1: FX985484) & $-(3)$ & None \\
\hline Homo sapiens (SOD3: NP 003093) & Secreted (2) & $1-18$ \\
\hline Mus musculus (SOD3: NP 035565) & Secreted (2) & $1-20$ \\
\hline Caenorhabditis elegans (SOD4: NP 001255003) & Secreted (1) & $1-17$ \\
\hline Drosophila melanogaster (SOD3: NP 725046) & Secreted (1) & $1-18$ \\
\hline Zootermopsis nevadensis (SOD3: KDR22972) & Secreted (1) & $1-18$ \\
\hline Reticulitermes speratus (RsSOD3A: FX985481) & Secreted (1) & $1-18$ \\
\hline Reticulitermes speratus (RsSOD3B: FX985482) & Secreted (1) & $1-20$ \\
\hline Homo sapiens (SOD2: NP 001019636) & Mitochondrial (3) & $1-24$ \\
\hline Mus musculus (SOD2: NP 038699) & Mitochondrial (3) & $1-24$ \\
\hline Caenorhabditis elegans (SOD2: NP 492290) & Mitochondrial (4) & $1-24$ \\
\hline Caenorhabditis elegans (SOD3: NP 510764) & Mitochondrial (3) & $1-24$ \\
\hline Drosophila melanogaster (SOD2: NP 476925) & Mitochondrial (5) & $1-17$ \\
\hline Zootermopsis nevadensis (SOD2: KDR21306) & Mitochondrial (2) & $1-80$ \\
\hline Reticulitermes speratus (RsSOD2: FX985483) & Mitochondrial (3) & $1-79$ \\
\hline
\end{tabular}

aTargetP classifies proteins as "secreted," "mitochondrial," or "other" (represented by “-" in the table). The reliability class (RC) ranges from 1 to 5 , where a smaller number indicates a stronger prediction. RC is a measure of the difference (d) between the highest and the second highest output scores. There are 5 RCs, defined as follows: (1) $d>0.800$, (2) $0.800>d>0.600$, (3) $0.600>d>0.400$, (4) $0.400>d>0.200$, and (5) $0.200>d$.

extended period of strong selection that should promote long life for the reproductives (queens and kings). Therefore, termite reproductives may be suitable model organisms to investigate if SODs are proximate causes of longevity. Reticulitermes speratus is one of the most studied subterranean termites, with regard to its reproductive system [23], symbiotic system [24], pheromone communication [25, 26], and antioxidant system $[27,28]$. In particular, a previous study revealed that $R$. speratus produced numerous neotenic queens by an asexual queen succession system [29], which results in adequate sample numbers for several experiments. Hence, we selected the termite $R$. speratus as a model organism.

In this study, we investigated if high expression of SODs is associated with the extreme lifespan of the queens of $R$. speratus. First, we identified three $\mathrm{Cu} / \mathrm{Zn}-\mathrm{SOD}$ sequences and one Mn-SOD sequence from the RNA sequencing (RNA-seq) data in $R$. speratus. Then, we tested if the levels of SODs are higher in termite queens than in nonreproductive individuals.

\section{Materials and Methods}

2.1. Termites. Animal ethics committee approval was not required for this study, which used insect species. Eight colonies of the termite $R$. speratus (workers, soldiers, nymphs, and mature neotenic queens) were collected from the experimental forest of Yamaguchi University, which is part of Mt. Himeyama in Yamaguchi, western Japan. We used pooled samples from different colonies for each experiment, as described in Table S1. These insect samples were preserved at $-80^{\circ} \mathrm{C}$ until use.

2.2. Molecular Analysis of R. speratus SODs. The whole transcriptome of $R$. speratus was examined using nextgeneration RNA-seq technology in a previous study [26]. We obtained mRNA sequences of SOD genes from the transcriptome data by performing a BLAST search with the amino acid sequences of translated SOD genes in the termite Zootermopsis nevadensis and other model organisms (Table S2). The presence and location of signal peptide cleavage sites in SOD proteins were predicted using the SignalP 4.1 server [30] and TargetP 1.1 server $[31,32]$. We performed multiple amino acid sequence alignments with CLUSTALW and conducted phylogenetic analyses using the molecular evolutionary genetics analysis software MEGA7 [33]. Gene evolutionary history was inferred using the maximum likelihood method based on the Whelan and Goldman model [34], which is the best model based on the Bayesian information criterion.

2.3. Protein Extraction. Whole insect body samples that had been stored at $-80^{\circ} \mathrm{C}$ were ground to powder in liquid nitrogen, then homogenised by sonication in tubes with $20 \mathrm{mM}$ Tris- $\mathrm{HCl}$ containing $2 \%$ protease inhibitor cocktail $(v / v)$, and followed by centrifugation at $17,000 \times \mathrm{g}$ for $10 \mathrm{~min}$ at $4^{\circ} \mathrm{C}$. The supernatants containing proteins were 


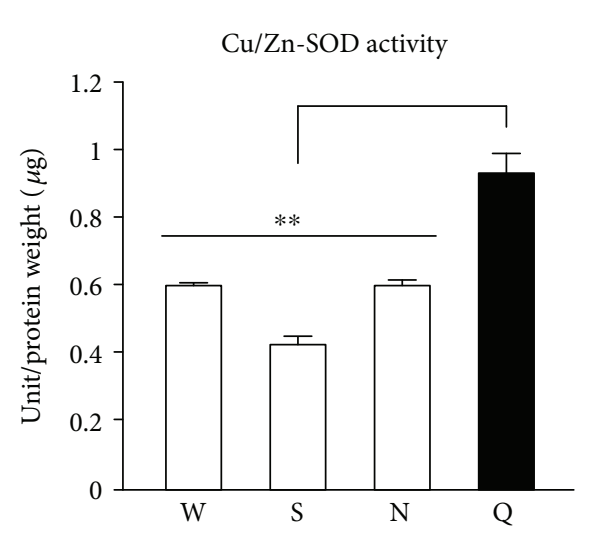

(a)

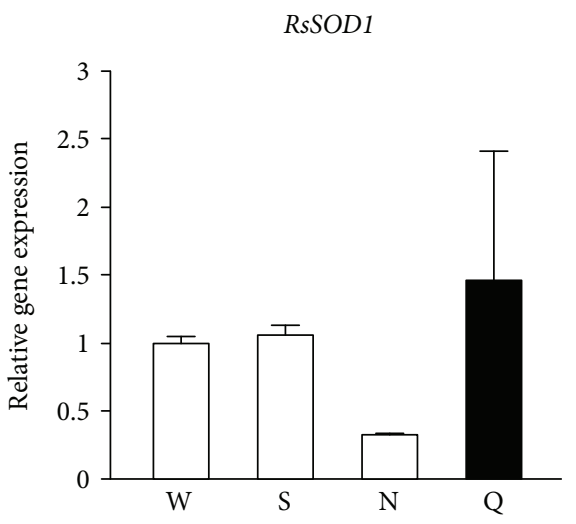

(b)

RsSOD3A

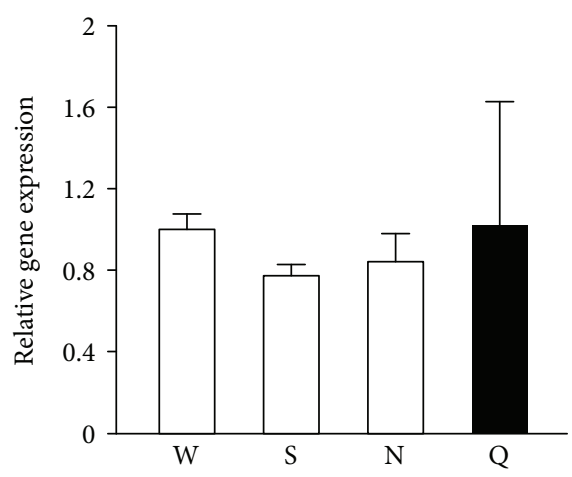

(c)

FIGURE 2: Cu/Zn-SOD activity and gene expression in R. speratus queens and nonreproductive individuals. (a) Queens ( $n=9)$ showed higher $\mathrm{Cu} / \mathrm{Zn}$-SOD activity than nonreproductive workers $(n=6 ; P=0.001)$, soldiers $(n=6 ; P<0.001)$, and nymphs $(n=6 ; P=0.001)$. (b) Queens $(n=9)$ did not have higher levels of RsSOD1 gene expression than workers $(n=12 ; P=1.000)$, soldiers $(n=12 ; P=1.000)$, or nymphs $(n=12 ; P=1.000)$. (c) Queens $(n=9)$ did not have higher levels of RsSOD3A gene expression than workers $(n=12 ; P=1.000)$, soldiers $(n=12 ; P=1.000)$, or nymphs $(n=12 ; P=1.000)$. We used pooled samples, as described in Table S1, for several replications. White and black bars indicate nonreproductive individuals and queens, respectively. Error bars represent SEM. Significance was measured by unpaired $t$-test followed by Holm's adjustment $\left({ }^{* *} \mathrm{P}<0.01\right)$. W: workers; S: soldiers; N: nymphs; Q: queens.

transferred to new tubes and used as samples for analyses. For each sample, protein concentration was measured with a bicinchoninic acid assay kit before extraction. The protein samples were preserved at $-80^{\circ} \mathrm{C}$ until use in antioxidant activity assays.

2.4. SOD Activity Assays. The activities of antioxidant enzymes were determined as in a previous report [35]. Briefly, we quantified SOD activity by using 2-(-4-iodophenyl)-3-(4-nitrophenyl)-5-(2,4-disulfophenyl)-2H-tetrazo-

lium (WST-1; Dojindo) to detect superoxide anion radicals. The reaction mixture contained diluted xanthine oxidase (approximately 0.2 units), $0.1 \mathrm{mM}$ xanthine, $25 \mu \mathrm{M}$ WST-1, $0.1 \mathrm{mM}$ EDTA, and $50 \mathrm{mM} \mathrm{Na}_{2} \mathrm{CO}_{3}(\mathrm{pH} 10.2)$ in a total volume of $3 \mathrm{~mL}$. The absorbance at $438 \mathrm{~nm}$ was monitored at $30^{\circ} \mathrm{C}$ for $1 \mathrm{~min}$. One unit was defined as the amount of enzyme required to inhibit $50 \%$ of an absorbance change of 0.06 per minute, which was equivalent to 0.8 units as determined by the standard procedure using cytochrome $\mathrm{c}$. Mn-SOD activities were defined as $1 \mathrm{mM} \mathrm{NaCN-resistant}$ activity. We performed 6-9 biological replicates for queens and nonreproductive individuals of $R$. speratus.

2.5. Measurement of Copper Concentrations. Copper $\left(\mathrm{Cu}^{2+}\right.$ and $\mathrm{Cu}^{+}$), which is a cofactor of $\mathrm{Cu} / \mathrm{Zn}-\mathrm{SOD}$, was extracted from termite queens and workers with $0.1 \mathrm{M} \mathrm{HCl}$ and measured using Metallo Assay Copper LS (Metallogenics) according to the accompanying manual. The measurements were based on the DiBr-PAESA method. The concentrations of copper were calculated as the molecular weights of copper relative to the protein weights. Sixteen biological replicates were performed, each with a queen and four workers.

2.6. Quantitative Real-Time PCR. We designed primer pairs for each the SOD genes using Primer3 (version 1.1.4; [36]; Table S3). Using ISOGEN reagent (Nippon Gene), total RNA was extracted individually from the whole bodies of termite workers, soldiers, nymphs, and queens that had been frozen with liquid nitrogen and stored at $-80^{\circ} \mathrm{C}$ until extraction. Immediately following extraction, cDNA was synthesized from the RNA using a PrimeScript ${ }^{\mathrm{TM}}$ RT reagent kit 
(Takara) and preserved at $-20^{\circ} \mathrm{C}$. Quantitative real-time PCR (qRT-PCR) was performed using a LightCycler ${ }^{\circledR}$ (Roche) with QuantiTect ${ }^{\circledR}$ SYBR $^{\circledR}$ Green PCR reagents (Qiagen). All procedures were performed in accordance with each manufacturer's protocol. GAPDH was selected as the reference gene. Relative expression levels were calculated using a typical $\Delta \Delta \mathrm{Ct}$ method. We performed 9-12 biological replicates for queens and nonreproductive individuals of $R$. speratus.

2.7. Statistical Analysis. R software package (version 3.2.2) was used for all statistical analyses. We performed unpaired $t$-tests followed by $P$ value corrections using Holm's method for multiple comparisons. All data in graphs are presented as the mean \pm standard error of the mean (SEM), and all calculated $P$ values are provided in the figure legends. Differences were considered significant when the $P$ value was $<0.05$.

\section{Results}

3.1. Identification of Cu/Zn-SOD and Mn-SOD Sequences in $R$. speratus. We previously examined the whole transcriptome of $R$. speratus using next-generation RNA-seq technology [26]. Via a subsequent homology search of the known amino acid sequence data from the termite $Z$. nevadensis and several model insects, we inferred the existence of three $\mathrm{Cu} / \mathrm{Zn}$-SOD sequences and one Mn-SOD sequence in the R. speratus transcriptome (Table S2). We determined, by sequence alignments with the well-characterized SOD1 and SOD3 of Homo sapiens, Mus musculus, C. elegans, and $D$. melanogaster, that the $R$. speratus $\mathrm{Cu} / \mathrm{Zn}-\mathrm{SOD}$ had conserved all of the active sites that are essential for metal binding and disulphide formation [37], suggesting that they are active $\mathrm{Cu} / \mathrm{Zn}$-SODs (Figure 1 ). We also confirmed that the $R$. speratus $\mathrm{Mn}$-SOD has a conserved manganesebinding site (Figure S1). Previous reports concluded that insects and other organisms have cytoplasmic and extracellular SODs [10, 38]. Our phylogenetic analysis revealed that one of the $R$. speratus sequences clustered with the cytoplasmic $\mathrm{Cu} / \mathrm{Zn}$-SODs of other organisms, whereas the other two sequences clustered with extracellular SODs (Figure S2). Furthermore, we determined that the predicted extracellular sequences have signal peptide cleavage sites (Figure 1 and Table 1). Although these phylogenetic analyses indicated that $R$. speratus has three $\mathrm{Cu} / \mathrm{Zn}-\mathrm{SOD}$, we discarded RsSOD3B (FX985482) because of low reliability, based on the high Evalue even in comparison to $Z$. nevadensis (Table S2). Thus, we treated RsSOD1 (FX985484) as the cytoplasmic Cu/ZnSOD gene, RsSOD3A (FX985481) as the extracellular Cu/ Zn-SOD gene, and RsSOD2 (FX985483) as the Mn-SOD gene in our analysis.

3.2. Termite Queens Have High Cu/Zn-SOD Activity in Comparison with Nonreproductive Individuals. Previously, we determined that $R$. speratus queens showed markedly lower levels of oxidative damage in comparison with nonreproductive workers, which is partly due to high catalase and peroxiredoxin expression and activity [27]. Therefore, we investigated if termite queens had higher SOD activities

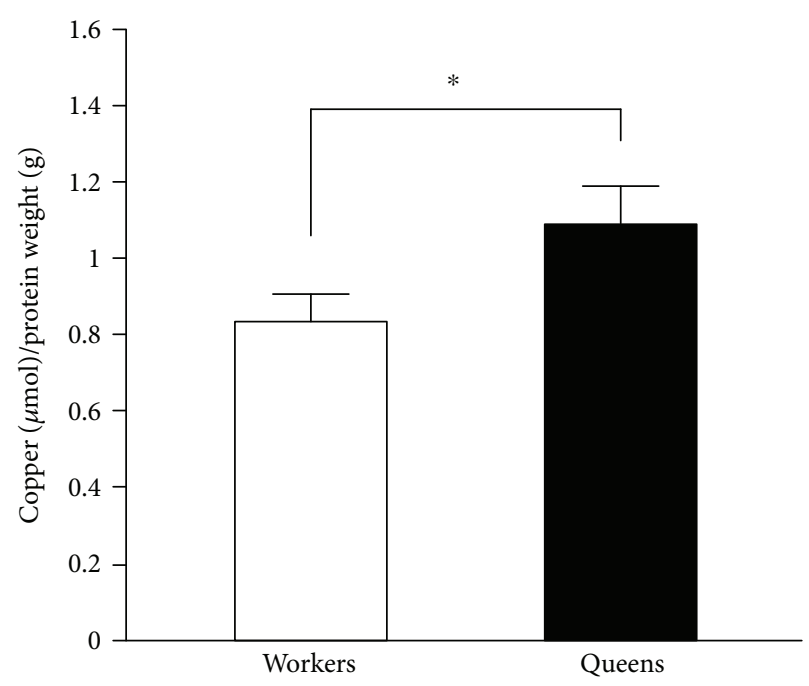

FIgURE 3: Measurement of copper concentrations in $R$. speratus queens and workers. Queens $(n=16)$ had significantly higher concentrations of copper than workers $(n=16 ; P=0.038)$. We used pooled samples, as described in Table S1, for several replications. White and black bars indicate workers and queens, respectively. Error bars represent SEM. Significance was measured by unpaired $t$-test $\left({ }^{*} P<0.05\right)$.

than nonreproductive individuals. We showed that $\mathrm{Cu} / \mathrm{Zn}$ SOD activity in $R$. speratus queens was higher than in nonreproductive individuals (Figure 2(a)). However, we found no differences in the expression of RsSOD1 and RsSOD3A, which encode cytoplasmic $\mathrm{Cu} / \mathrm{Zn}-\mathrm{SOD}$ and extracellular $\mathrm{Cu} / \mathrm{Zn}-\mathrm{SOD}$, respectively, between the queens and nonreproductive individuals (Figures 2(b) and 2(c)). We next examined if the copper concentrations corresponded to the $\mathrm{Cu}$ / $\mathrm{Zn}$-SOD activity in termites. We found that termite queens have higher copper concentrations than nonreproductive workers (Figure 3). On the other hand, the Mn-SOD activity in $R$. speratus queens differed only from that in soldiers (Figure 4(a)). In addition, the queens and nonreproductive individuals had similar levels of RsSOD2 expression (Figure 4(b)).

\section{Discussion}

To date, the determining factors of organismal longevity are among the most fascinating problems for many scientists. Eusocial insects have drawn attention as models for the study of the roles of specific genes in the aging process [9]. In the present study, we found that termite queens have higher $\mathrm{Cu} / \mathrm{Zn}-\mathrm{SOD}$ activity than nonreproductive individuals in the subterranean termite $R$. speratus. Given the long lifespan of termite queens, these findings suggest that high $\mathrm{Cu} / \mathrm{Zn}$ SOD activity may contribute to longevity. Our findings contract with those of earlier studies of eusocial Hymenoptera, which suggested that queen longevity was not associated with cytoplasmic Cu/Zn-SOD activity $[39,40]$. In Hymenoptera, $\mathrm{Cu} / \mathrm{Zn}-\mathrm{SOD}$ is reportedly highly expressed in venom glands [41] and secreted in venom [38]. This unique trait may obscure a relationship between SOD levels and longevity. Although eusocial insects have attracted considerable 


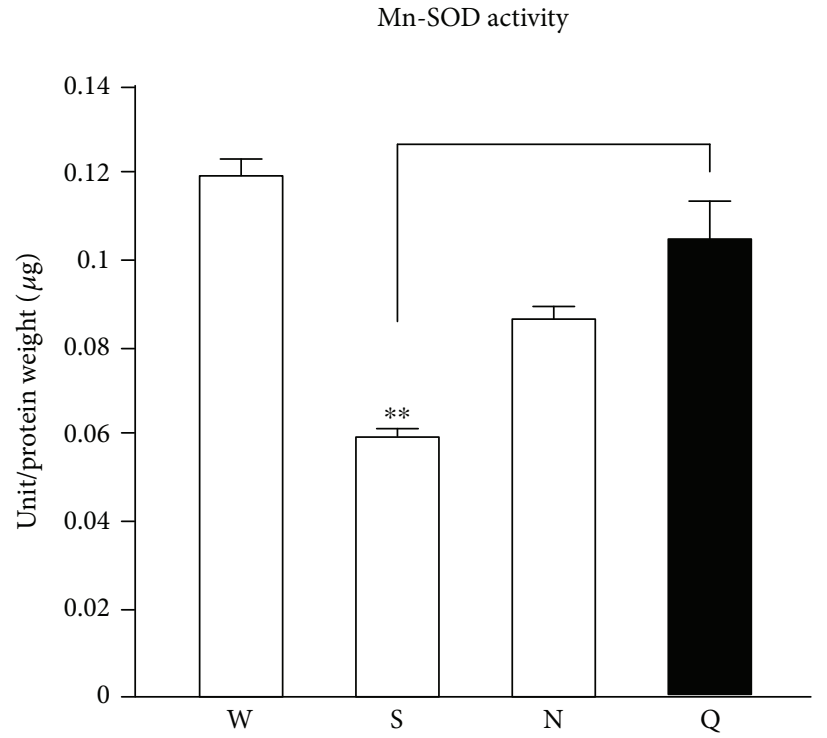

(a)

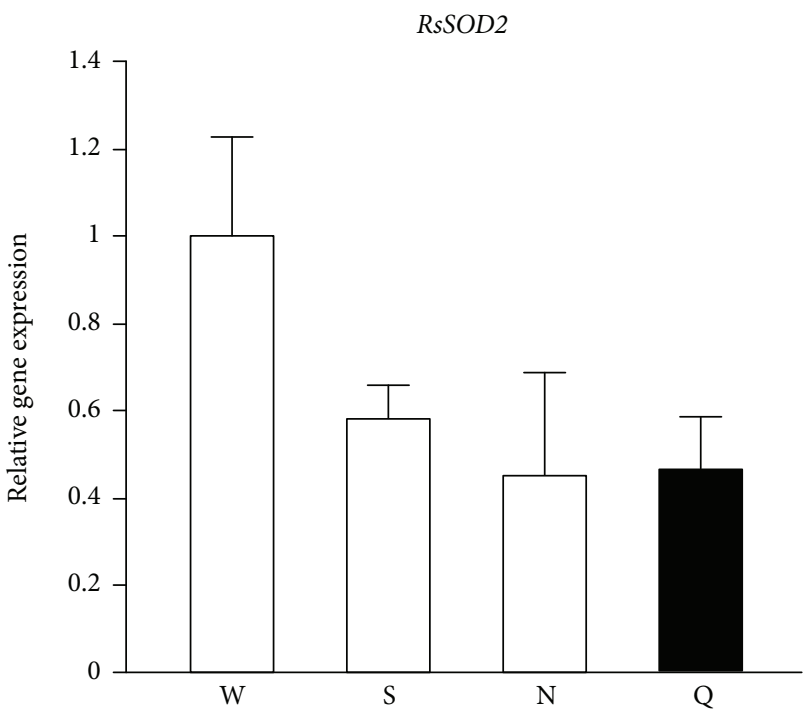

(b)

FIGURE 4: Mn-SOD activity and gene expression in R. speratus queens and nonreproductive individuals. (a) Queens ( $n=9)$ had higher MnSOD activity than soldiers $(n=6 ; P=0.004)$ but not workers $(n=6 ; P=0.214)$ or nymphs $(n=6 ; P=0.133)$. (b) Queens $(n=9)$ did not have higher levels of RsSOD2 gene expression than workers $(n=12 ; P=0.224)$, soldiers $(n=12 ; P=0.813)$, or nymphs $(n=12 ; P=0.960)$. We used pooled samples, as described in Table S1, for several replications. White and black bars indicate nonreproductive individuals and queens, respectively. Error bars represent SEM. Significance was measured by unpaired $t$-test followed by Holm's adjustment $\left({ }^{* *} P<0.01\right)$. W: workers; S: soldiers; N: nymphs; Q: queens.

attention for aging research, termites have not yet been investigated. This study highlights the importance of studying eusocial insects like termites, in addition to Hymenoptera, in the search for the mechanisms that contribute to the extraordinarily long lifespan of insect queens.

Oral fluid exchange (trophallaxis) is important for nutritional dynamics and communication in termites because queens depend on nestmate workers for most nutrients. Interestingly, we found that termite queens have higher copper concentrations than workers. Subterranean termites acquire several metal ions, including copper, from the soil and other foods [42]. This implies that higher copper concentrations in termite queens occur by trophic accumulation in castes. High copper concentrations are associated with increased $\mathrm{Cu} / \mathrm{Zn}$-SOD activity [43]. In the present study, we demonstrated that there were no significant differences in RsSOD1 and RsSOD3A expression among the termite castes although the queens had higher $\mathrm{Cu} / \mathrm{Zn}$-SOD activity than nonreproductive individuals. There are often discrepancies between enzyme activity and gene expression levels. A previous report indicated that copper is critical for $\mathrm{Cu} /$ Zn-SOD activity and modulates enzymatic activity in the absence of changes in gene expression [43]. Therefore, the difference in copper concentrations between the queens and workers may be critical for their disparate levels of $\mathrm{Cu}$ / Zn-SOD activity. These results indicate that trophallaxis (from workers to queens) not only reduces the energy cost of foraging in termite queens but also provides nutritional benefits to the queens. Thus, trophallaxis may play an important role in nutrient accumulation contributing to termite queen longevity.
It remains unclear if increases in $\mathrm{Cu} / \mathrm{Zn}$-SOD activity are localized to specific tissues in termite queens. In mammals, ROS and SODs are thought to play important roles in several aspects of reproductive physiology because superoxide is generated and SOD is expressed in the ovary [44]. Additionally, several studies have reported a relationship between reproduction and antioxidant systems in insects [45-47]. Therefore, $\mathrm{Cu} / \mathrm{Zn}-\mathrm{SOD}$ may have an important role to play in reproductive processes, as well as in longevity, in termites. These findings suggest that $\mathrm{Cu} / \mathrm{Zn}$-SOD activity may be higher in reproductive tissues than in other somatic tissues. To determine if $\mathrm{Cu} / \mathrm{Zn}$-SOD is associated with reproduction in termites, future studies featuring tissue-specific analyses of $\mathrm{Cu} / \mathrm{Zn}-\mathrm{SOD}$ are required. Moreover, it is estimated that copper tends to accumulate in the reproductive tissues in termite queens. It is also necessary to determine the mechanisms by which copper transfer and accumulation occur in termite.

\section{Conflicts of Interest}

The authors declare no competing financial interests.

\section{Authors' Contributions}

Eisuke Tasaki and Yoshihito Iuchi conceived and designed the study. Eisuke Tasaki, Kazuya Kobayashi, and Kenji Matsuura collected the biological material. Kazuya Kobayashi performed RNA-seq analysis and Eisuke Tasaki constructed the phylogenetic tree. Eisuke Tasaki performed the enzymatic activity assays, qRT-PCR, and other biochemical analyses. Eisuke Tasaki drafted the manuscript 
with contributions from Kazuya Kobayashi, Kenji Matsuura, and Yoshihito Iuchi.

\section{Acknowledgments}

This work was supported by Japan Society for the Promotion of Science Grant numbers 26660113 to Yoshihito Iuchi and 25221206 to Kenji Matsuura.

\section{Supplementary Materials}

Supplementary 1. Figure S1: alignment and structure conservation of Mn-SOD sequences. Multiple sequence alignment of Homo sapiens, Mus musculus, Caenorhabditis elegans, Drosophila melanogaster, Zootermopsis nevadensis, and Reticulitermes speratus. Conserved functional residues are highlighted in bold characters in grey (circled $\mathrm{M}=$ manganese-binding site).

Supplementary 2. Figure S2: maximum likelihood molecular phylogenetic tree of $\mathrm{Cu} / \mathrm{Zn}-\mathrm{SOD}$ sequences. We inferred the evolutionary history of the $\mathrm{Cu} / \mathrm{Zn}$-SODs using the maximum likelihood method based on the Whelan and Goldman model. The tree with the highest log likelihood $(-2592.76)$ is shown. The percentage of trees in which the associated taxa clustered together is shown above the branches. Initial tree(s) for the heuristic search were obtained automatically by applying Neighbor-Joining and BioNJ algorithms to a matrix of pairwise distances estimated using a JTT model and then selecting the topology with a superior log likelihood value. A discrete gamma distribution was used to model the evolutionary rate differences among the sites ( 5 categories $[+G$, parameter $=1.8410]$ ). The rate variation model allowed for some sites to be evolutionarily invariable ([+/], $21.40 \%$ sites). The tree is drawn to scale, with branch lengths measured in the number of substitutions per site. The analysis involved 14 amino acid sequences. All positions containing gaps and missing data were eliminated. There were a total of 137 positions in the final dataset. Evolutionary analyses were conducted in MEGA7.

Supplementary 3. Table S1: termite samples.

Supplementary 4. Table S2: target gene details.

Supplementary 5. Table S3: primer sequences.

\section{References}

[1] S. Jemielity, M. Chapuisat, J. D. Parker, and L. Keller, "Long live the queen: studying aging in social insects," Age, vol. 27, no. 3, pp. 241-248, 2005.

[2] L. Keller and M. Genoud, "Extraordinary lifespans in ants: a test of evolutionary theories of ageing," Nature, vol. 389, no. 6654, pp. 958-960, 1997.

[3] R. E. Page Jr and C. Y.-S. Peng, "Aging and development in social insects with emphasis on the honey bee, Apis mellifera L," Experimental Gerontology, vol. 36, no. 4-6, pp. 695-711, 2001.

[4] L. Partridge, D. Gems, and D. J. Withers, "Sex and death: what is the connection?," Cell, vol. 120, no. 4, pp. 461-472, 2005.
[5] O. R. Jones, A. Scheuerlein, R. Salguero-Gómez et al., "Diversity of ageing across the tree of life," Nature, vol. 505, no. 7482, pp. 169-173, 2014.

[6] C. M. Sgrò and L. Partridge, "A delayed wave of death from reproduction in Drosophila," Science, vol. 286, no. 5449, pp. 2521-2524, 1999.

[7] H. Hsin and C. Kenyon, "Signals from the reproductive system regulate the lifespan of C. elegans," Nature, vol. 399, no. 6734, pp. 362-366, 1999.

[8] J. Heinze and A. Schrempf, “Terminal investment: individual reproduction of ant queens increases with age," PLoS One, vol. 7, no. 4, article e35201, 2012.

[9] L. Keller and S. Jemielity, "Social insects as a model to study the molecular basis of ageing," Experimental Gerontology, vol. 41, no. 6, pp. 553-556, 2006.

[10] J. D. Parker, K. M. Parker, and L. Keller, "Molecular phylogenetic evidence for an extracellular $\mathrm{Cu} \mathrm{Zn}$ superoxide dismutase gene in insects," Insect Molecular Biology, vol. 13, no. 6, pp. 587-594, 2004.

[11] I. N. Zelko, T. J. Mariani, and R. J. Folz, "Superoxide dismutase multigene family: a comparison of the CuZn-SOD (SOD1), $\mathrm{Mn}-\mathrm{SOD}$ (SOD2), and EC-SOD (SOD3) gene structures, evolution, and expression," Free Radical Biology \& Medicine, vol. 33, no. 3, pp. 337-349, 2002.

[12] W. Dröge, "Free radicals in the physiological control of cell function," Physiological Reviews, vol. 82, no. 1, pp. 47-95, 2002.

[13] T. Finkel and N. J. Holbrook, "Oxidants, oxidative stress and the biology of ageing," Nature, vol. 408, no. 6809, pp. 239247, 2000.

[14] W. Orr and R. Sohal, "Extension of life-span by overexpression of superoxide dismutase and catalase in Drosophila melanogaster," Science, vol. 263, no. 5150, pp. 1128-1130, 1994.

[15] T. L. Parkes, A. J. Elia, D. Dickinson, A. J. Hilliker, J. P. Phillips, and G. L. Boulianne, "Extension of Drosophila lifespan by overexpression of human SOD1 in motorneurons," Nature Genetics, vol. 19, no. 2, pp. 171-174, 1998.

[16] J. Sun and J. Tower, "FLP recombinase-mediated induction of $\mathrm{Cu} / \mathrm{Zn}$-superoxide dismutase transgene expression can extend the life span of adult Drosophila melanogaster flies," Molecular and Cellular Biology, vol. 19, no. 1, pp. 216-228, 1999.

[17] P. Fabrizio, L. L. Liou, V. N. Moy et al., "SOD2 functions downstream of Sch9 to extend longevity in yeast," Genetics, vol. 163, no. 1, pp. 35-46, 2003.

[18] S. Melov, J. Ravenscroft, S. Malik et al., "Extension of life-span with superoxide dismutase/catalase mimetics," Science, vol. 289, no. 5484, pp. 1567-1569, 2000.

[19] M. Keaney and D. Gems, "No increase in lifespan in Caenorhabditis elegans upon treatment with the superoxide dismutase mimetic EUK-8," Free Radical Biology \& Medicine, vol. 34, no. 2, pp. 277-282, 2003.

[20] T. T. Huang, E. J. Carlson, A. M. Gillespie, Y. Shi, and C. J. Epstein, "Ubiquitous overexpression of $\mathrm{CuZn}$ superoxide dismutase does not extendlife span in mice," The Journals of Gerontology: Series A, Biological Sciences and Medical Sciences, vol. 55, no. 1, pp. B5-B9, 2000.

[21] N. O. Seto, S. Hayashi, and G. M. Tener, "Overexpression of $\mathrm{Cu}-\mathrm{Zn}$ superoxide dismutase in Drosophila does not affect life-span," Proceedings of the National Academy of Sciences of the United States of America, vol. 87, no. 11, pp. 4270-4274, 1990. 
[22] W. C. Orr, R. J. Mockett, J. J. Benes, and R. S. Sohal, "Effects of overexpression of copper-zinc and manganese superoxide dismutases, catalase, and thioredoxin reductase genes on longevity in Drosophila melanogaster," The Journal of Biological Chemistry, vol. 278, no. 29, pp. 26418-26422, 2003.

[23] K. Matsuura, "Evolution of the asexual queen succession system and its underlying mechanisms in termites," The Journal of Experimental Biology, vol. 220, no. 1, pp. 63-72, 2017.

[24] M. Ohkuma, "Termite symbiotic systems: efficient biorecycling of lignocellulose," Applied Microbiology and Biotechnology, vol. 61, no. 1, pp. 1-9, 2003.

[25] K. Matsuura, C. Himuro, T. Yokoi, Y. Yamamoto, E. L. Vargo, and L. Keller, "Identification of a pheromone regulating caste differentiation in termites," Proceedings of the National Academy of Sciences of the United States of America, vol. 107, no. 29, pp. 12963-12968, 2010.

[26] Y. Mitaka, K. Kobayashi, A. Mikheyev, M. M. Y. Tin, Y. Watanabe, and K. Matsuura, "Caste-specific and sexspecific expression of chemoreceptor genes in a termite," PLoS One, vol. 11, no. 1, article e0146125, 2016.

[27] E. Tasaki, K. Kobayashi, K. Matsuura, and Y. Iuchi, "An efficient antioxidant system in a long-lived termite queen," PLoS One, vol. 12, no. 1, article e0167412, 2017.

[28] E. Tasaki, H. Sakurai, M. Nitao, K. Matsuura, and Y. Iuchi, "Uric acid, an important antioxidant contributing to survival in termites," PLoS One, vol. 12, no. 6, article e0179426, 2017.

[29] K. Matsuura, E. L. Vargo, K. Kawatsu et al., "Queen succession through asexual reproduction in termites," Science, vol. 323, no. 5922 , p. $1687,2009$.

[30] T. N. Petersen, S. Brunak, G. von Heijne, and H. Nielsen, "SignalP 4.0: discriminating signal peptides from transmembrane regions," Nature Methods, vol. 8, no. 10, pp. 785-786, 2011.

[31] O. Emanuelsson, H. Nielsen, S. Brunak, and G. von Heijne, "Predicting subcellular localization of proteins based on their N-terminal amino acid sequence," Journal of Molecular Biology, vol. 300, no. 4, pp. 1005-1016, 2000.

[32] H. Nielsen, J. Engelbrecht, S. Brunak, and G. von Heijne, "Identification of prokaryotic and eukaryotic signal peptides and prediction of their cleavage sites," Protein Engineering, vol. 10, no. 1, pp. 1-6, 1997.

[33] K. Tamura, J. Dudley, M. Nei, and S. Kumar, "MEGA4: molecular evolutionary genetics analysis (MEGA) software version 4.0," Molecular Biology and Evolution, vol. 24, no. 8, pp. 1596-1599, 2007.

[34] S. Whelan and N. Goldman, "A general empirical model of protein evolution derived from multiple protein families using a maximum-likelihood approach," Molecular Biology and Evolution, vol. 18, no. 5, pp. 691-699, 2001.

[35] Y. Iuchi, F. Okada, K. Onuma et al., "Elevated oxidative stress in erythrocytes due to a SOD1 deficiency causes anaemia and triggers autoantibody production," Biochemical Journal, vol. 402, no. 2, pp. 219-227, 2007.

[36] S. Rozen and H. Skaletsky, "Primer3 on the WWW for general users and for biologist programmers," Methods in Molecular Biology, vol. 132, pp. 365-386, 2000.

[37] J. J. P. Perry, D. S. Shin, E. D. Getzoff, and J. A. Tainer, "The structural biochemistry of the superoxide dismutases," Biochimica et Biophysica Acta (BBA) - Proteins and Proteomics, vol. 1804, no. 2, pp. 245-262, 2010.
[38] D. Colinet, D. Cazes, M. Belghazi, J. L. Gatti, and M. Poirié, "Extracellular superoxide dismutase in insects: characterization, function, and interspecific variation in parasitoid wasp venom," The Journal of Biological Chemistry, vol. 286, no. 46, pp. 40110-40121, 2011.

[39] J. D. Parker, K. M. Parker, B. H. Sohal, R. S. Sohal, and L. Keller, "Decreased expression of $\mathrm{Cu}-\mathrm{Zn}$ superoxide dismutase 1 in ants with extreme lifespan," Proceedings of the National Academy of Sciences of the United States of America, vol. 101, no. 10, pp. 3486-3489, 2004.

[40] M. Corona, K. A. Hughes, D. B. Weaver, and G. E. Robinson, "Gene expression patterns associated with queen honey bee longevity," Mechanisms of Ageing and Development, vol. 126, no. 11, pp. 1230-1238, 2005.

[41] N. Peiren, D. C. de Graaf, F. Vanrobaeys et al., "Proteomic analysis of the honey bee worker venom gland focusing on the mechanisms of protection against tissue damage," Toxicon, vol. 52 , no. 1 , pp. $72-83,2008$.

[42] M. P. Janzow and T. M. Judd, “The termite Reticulitermes flavipes (Rhinotermitidae: Isoptera) can acquire micronutrients from soil," Environmental Entomology, vol. 44, no. 3, pp. 814-820, 2015.

[43] J. L. Frendo, P. Thérond, J. Guibourdenche, J. M. Bidart, M. Vidaud, and D. Evain-Brion, "Modulation of copper/zinc superoxide dismutase expression and activity with in vitro differentiation of human villous cytotrophoblasts," Placenta, vol. 21, no. 8, pp. 773-781, 2000.

[44] N. Sugino, "Reactive oxygen species in ovarian physiology," Reproductive Medicine and Biology, vol. 4, pp. 31-44, 2005.

[45] V. Michalkova, J. B. Benoit, G. M. Attardo, J. Medlock, and S. Aksoy, "Amelioration of reproduction-associated oxidative stress in a viviparous insect is critical to prevent reproductive senescence," PLoS One, vol. 9, no. 4, article e87554, 2014.

[46] R. J. DeJong, L. M. Miller, A. Molina-Cruz, L. Gupta, S. Kumar, and C. Barillas-Mury, "Reactive oxygen species detoxification by catalase is a major determinant of fecundity in the mosquito Anopheles gambiae," Proceedings of the National Academy of Sciences of the United States of America, vol. 104, no. 7, pp. 2121-2126, 2007.

[47] H. Diaz-Albiter, R. Mitford, F. A. Genta, M. R. V. Sant'Anna, and R. J. Dillon, "Reactive oxygen species scavenging by catalase is important for female Lutzomyia longipalpis fecundity and mortality," PLoS One, vol. 6, no. 3, article e17486, 2011. 


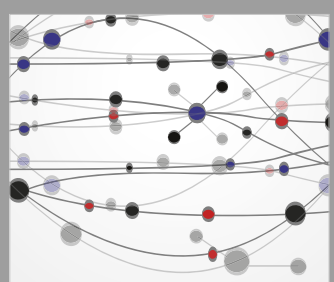

The Scientific World Journal
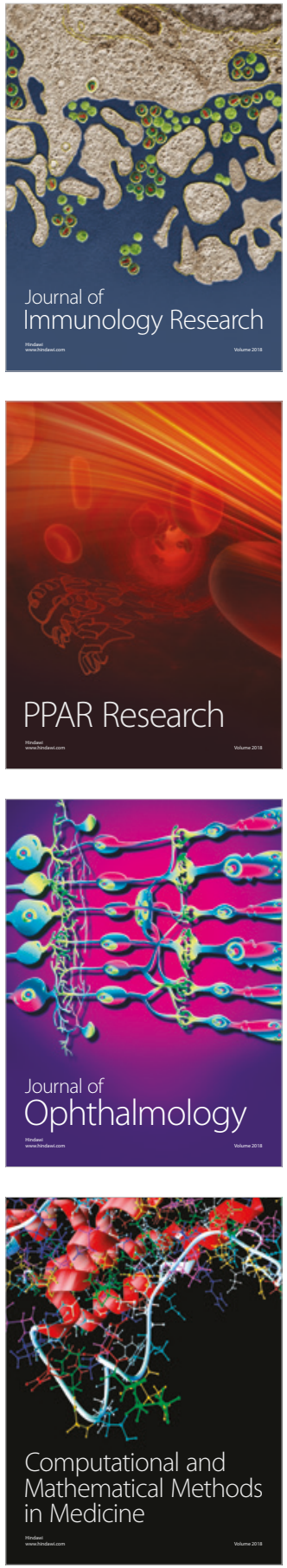

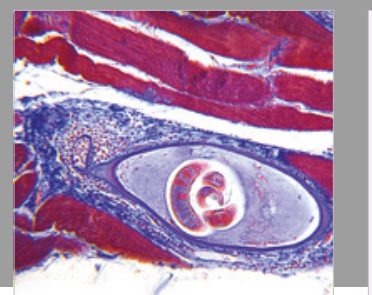

Gastroenterology Research and Practice

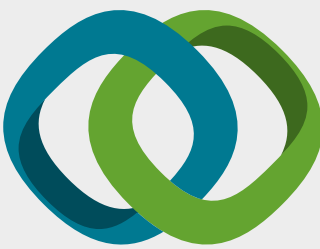

\section{Hindawi}

Submit your manuscripts at

www.hindawi.com
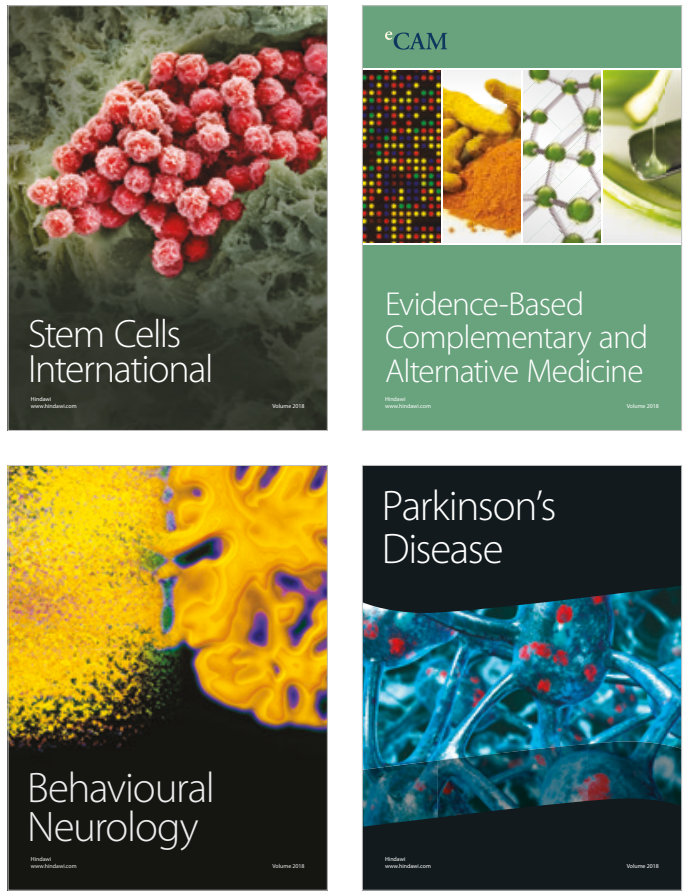

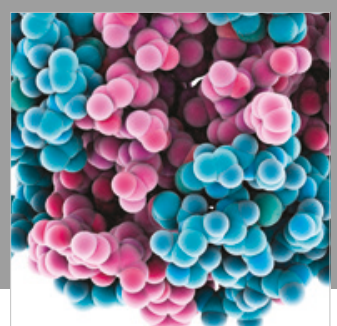

ournal of

Diabetes Research

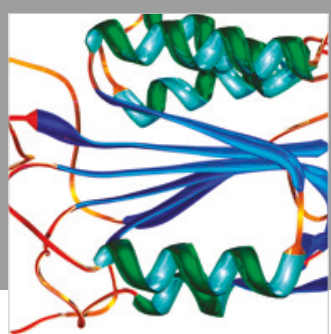

Disease Markers
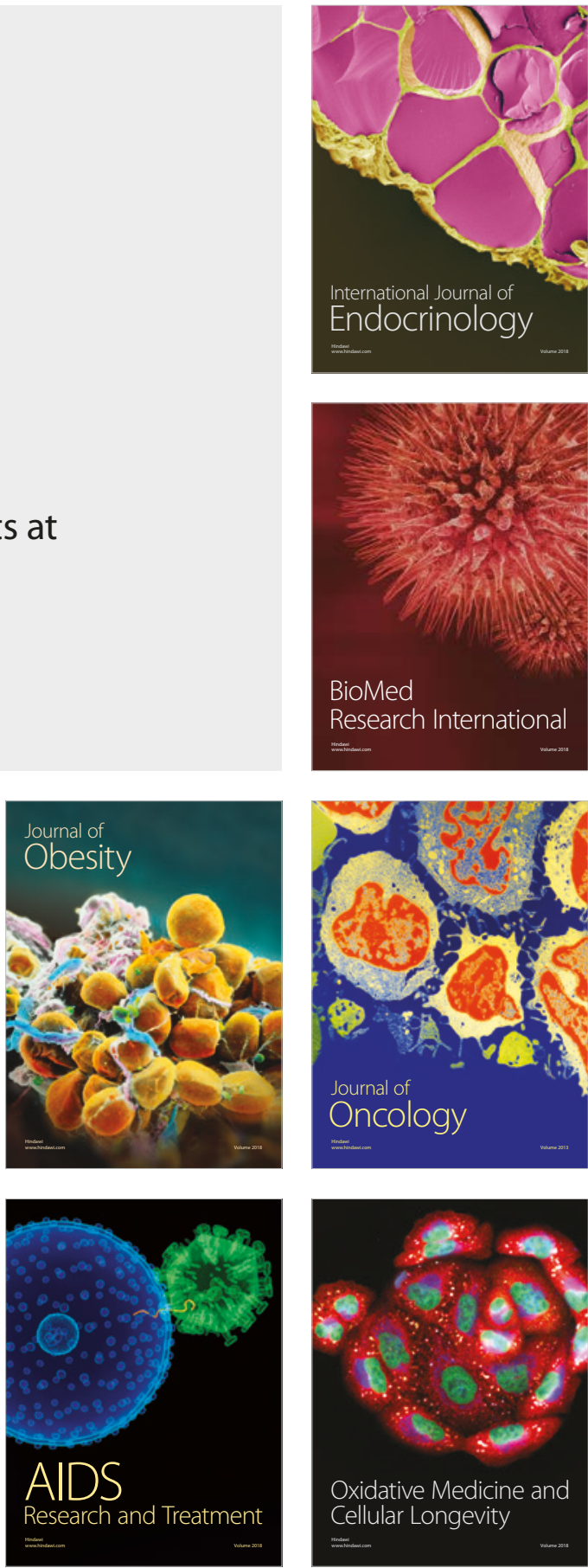